\title{
The Inflammatory Contracted Bladder
}

\author{
Hector Cantu ${ }^{1}$ - Siti Nur Masyithah Maarof ${ }^{1} \cdot$ Hashim Hashim ${ }^{1,2,3}$
}

Published online: 22 April 2019

(C) The Author(s) 2019

\begin{abstract}
Purpose of Review This review will present the inflammatory contracted bladder as a clinical entity and will address its pathophysiology, diagnosis and treatment.

Recent Findings The inflammatory contracted bladder is relevant since it is not a recognised urological condition and it can be found in several affections to the urinary tract. Its medical management depends on its aetiology and severity.

Summary The inflammatory contracted bladder (ICB) is an ambiguous term that is used to describe the morphology of the inflamed bladder with a reduced volumetric capacity and compliance. This condition is found in some urological pathologies such as bladder outlet obstruction, infections, drug toxicity, radiation cystitis and other inflammatory diseases like interstitial cystitis, malacoplakia and eosinophilic cystitis. This entity is usually presented at the end stage of disease and represents an irreversible damage to the urinary tract.
\end{abstract}

Keywords Inflammatory contracted bladder $\cdot$ Volumetric capacity $\cdot$ Compliance

\section{Introduction}

The term inflammatory contracted bladder (ICB) is included in a diverse spectrum of urological diseases that affects the function of the bladder, its volumetric capacity and compliance; this condition could also cause deterioration of the upper urinary tract and renal impairment. The presentation of the ICB involves a wide range of storage and voiding symptoms. There is considerable ambiguity about the aetiology, morphology and clinical significance of the contracted bladder; however, it is important to assess and treat this condition because of the potential irreversible damage to the urinary tract.

This article is part of the Topical Collection on Inflammatory/Infectious Bladder Disorders

Hashim Hashim

h.hashim@gmail.com

Hector Cantu

jesushectorcantu@gmail.com

Siti Nur Masyithah Maarof

snmasyithah@gmail.com

1 Bristol Urological Institute, Bristol, UK

2 Urodynamic Unit Southmead Hospital, Westbury-on-Trym, Bristol BS10 5NB, UK

3 University of Bristol, Senate House, Tyndall Ave, Bristol BS8 1TH, UK

\section{Background}

Recent descriptions of the functional abnormalities of the bladder were initially described in the French literature in 1897 by Jean Casimir Félix Guyon [1]. During 1917 in San Francisco, Krotoszyner postulated the clinical diagnosis of the contracted bladder and reported some cystoscopic characteristics of this condition. He stated that the underlying causes of the contracted bladder may share a similar disordered physiological process; however, it may occur on the basis of a variety of vesical and extravesical factors [2]. A volumetric capacity of less than $50 \mathrm{~mL}$ was used as a reference.

The current standardisation of terminology of lower urinary tract function reported by the sub-committee of the International Continence Society, does not present the ICB as an associated condition with lower urinary tract dysfunction and urodynamic studies [3••]. This review presents a comprehensive analysis of the ICB according to the database findings in the medical literature.

\section{Pathophysiology}

Several theories could be postulated in the pathophysiology of the ICB. It is important to recognise that it is challenging to determine specific tissue changes because this condition 
involves several pathological pathways in the development of bladder dysfunction.

\section{Urothelium}

Alterations on the urothelial cells like the loss of tight junctions or modifications on the membrane cell structure are compromised by several conditions such as infections, inflammation, chemical exposure or radiation. The impairment of the epithelial impermeability could manifest with elevation of inflammatory and fibrosis markers together with reduced bladder capacity and ICB $[4,5 \bullet]$.

\section{Stroma}

The main elements of the bladder stroma are collagen and elastin surrounded by fibroblasts and a matrix of proteoglycans. The normal bladder responds well to changes in bladder volume and pressure. Bladder collagen and elastin are related to tissue compliance; there are several collagen isoforms, type I and III are the most important. An increased type III to I ratio has been reported in the non-compliant bladder tissue where there is an increased expression of type III collagen mRNA [6]. Elastin gene suppression has also been reported in the non-compliant human bladder [7].

\section{Hypertrophy}

Molecular alterations on the signalling pathways have been documented on animal models with bladder dysfunction. Decompensated bladders present smooth muscle hypertrophy and this change of morphology has an effect on urinary frequency [8]. A direct link between ICB and detrusor hypertrophy has not been documented. However, it can be hypothesised that ICB pathophysiology might share some biochemical cascades.

\section{Clinical Scenarios}

The ICB is a morphologic feature that can be presented in some stages of multiple urological conditions. Radiological characteristics include a reduced volumetric capacity, bladder wall thickening with or without associated vesicoureteral reflux. Cystoscopic findings (when possible to execute due to poor capacity) may differ, but mostly is a disturbed mucosa in the form of erythema, ulcers, petechial, haemorrhage, and trabeculations [2]. Table 1 summarises all the clinical scenarios in which the ICB can be recognised.

\section{Diagnosis and Investigations}

Basic foundation for subsequent evaluation can be obtained from medical history and physical examination. Factors such as medication use, specific past medical history such as tuberculosis, ketamine abuse or pelvic radiotherapy must also be factored into the decision and diagnostic paradigm [9]. Certain physical examination factors must also be evaluated such as enlarged prostate gland, hydronephrosis or any associated neurologic abnormalities.

Both men and women with history of lower urinary tract symptoms will require urinalysis [10]. Assessment modalities include estimation of bladder capacity, post void residual assessment, cystoscopy, radiographic techniques such as ultrasound of the urinary tract, CT urogram, voiding cystourethrogram (VCUG) and MRI of the bladder outlet and urethra can be performed. Further attempts at refining the diagnosis of ICB may require urodynamic assessment $[9,11]$.

Bladder capacity can be determined either from the maximum amount voided on the bladder diary, with non-invasive uroflow study or during urodynamics. Men and women require a quality of life questionnaire such as the ICIQ-LUTS questionnaire. Men [10] can also fill the International Prostate Symptom Score (IPSS) questionnaire, have a digital rectal examination and serum prostate-specific antigen (PSA) analyses. In women [10], a pad test may be required if they complained of urinary incontinence.

To confirm tuberculosis infection as the cause of ICB, it is important to demonstrate mycobacterium in urine and radiographic examination [12]. 'Sterile pyuria' is not very sensitive or specific for urogenital tuberculosis, but persisting sterile pyuria in an individual at risk should increase the index of suspicion [13]. For the anatomical and functional details of kidneys and ureters, intravenous urography (IVU) has been considered to be one of the most useful tests. Pyelograms disclosed abnormalities in $94 \%$ of cases [14]. Unfortunately, pyelogram is useful only for late cavernous forms. If a patient has renal failure, MRI of the urinary tract can be used [12]. It may also be useful to perform provocation test with injection of 20,50 or 100 units of tuberculin subcutaneously [15]. Highly sensitive examinations such as radiometric liquid culture systems (i.e. BACTEC ${ }^{\circledR}$, Becton Dickinson, USA) and polymerase chain reaction (PCR) can also identify the mycobacterium [12].

Schistosomiasis, also known as bilharzia, is a common intravascular infection caused by the parasitic Schistosoma trematode worms which can predispose to ICB $[16,17]$. 'Sandy patches' which are areas of roughened bladder mucosa surrounding egg deposits are identified on cystoscopy examination. The trematode worm eggs are released in urine and are detected by microscopy in a urine sample. In cases where no egg is detectable in the urine, biopsy of the bladder may be 
Table 1 Clinical scenarios of the ICB

\begin{tabular}{ll}
\hline Clinical scenarios & Features that meet the criteria of ICB \\
\hline Bladder outlet obstruction & Decompensation stage \\
Congenital obstructed bladder & Delayed treatment \\
Neurogenic bladder & Deferred stages (e.g. spinal shock) \\
Infections & When source and type of infection compromises \\
Tuberculosis & the bladder, especially in advanced disease \\
Schistosomiasis & With positive cystoscopy and histology \\
Inflammatory conditions & Multifocal presentation \\
Interstitial cystitis & Fibrotic stage \\
Malacoplakia & ICB as part of natural history of Ketamine bladder syndrome \\
Eosinophilic cystitis & Toxicity from chemotherapy or immunotherapy is rare presentation, \\
Drugs and medications & evidence mostly presented as side effect and based on case reports \\
Ketamine & \\
Bacillus Calmette-Guérin (BCG) & \\
Mitomycin C & \\
Epirubicin & Associated with high dose of radiation \\
Gefitinib & When used for more than 6-12 months on free drainage \\
Radiation cystitis & Indwelling catheter
\end{tabular}

considered for diagnosis in patients with a typical clinical presentation of schistosomiasis.

ICB caused by inflammatory conditions will require a cystoscopy and bladder biopsy. Whereas ICB caused by drugs, medications and radiation cystitis can be determined during history taking. Urinalysis and urine culture in this group of ICB, typically show sterile urine and occasionally, sterile pyuria [18]. They will also complain of frequent voiding which indicate small bladder capacity. Therefore, a bladder diary should ideally be kept [19]. Radiographic investigations of the upper urinary tract may reveal unilateral or bilateral hydronephrosis (Fig. 1), circumferential mural thickening with a small bladder capacity (Fig. 2) [19]. Small bladder capacity (usually $<150 \mathrm{~mL}$ ) with decreased bladder compliance associated with detrusor overactivity will be apparent on video urodynamic [20, 21] (Fig. 3).

\section{Bladder Outlet Obstruction}

Patients with bladder outflow obstruction mostly because of benign prostatic obstruction (BPO) could present with detrusor hypertrophy, obstruction induced detrusor overactivity and alterations in detrusor contractility. BPO produces a structural damage on the bladder wall; this process could have several stages. Commencing with a compensated phase, in which the bladder counteracts the obstruction with a relatively constant bladder mass and detrusor contraction, the end stage or decompensation is characterised by a thick fibrous wall with a low volumetric capacity and poor compliance. At this stage, detrusor contraction is practically absent [9]. All of these characteristics describe the ICB; therefore, its treatment should be focused in managing bladder outflow obstruction at an early stage.

\section{Congenital Obstructed Bladder}

Bladders that are defunctionalized early in life because of congenital obstruction like posterior urethral valves, result in

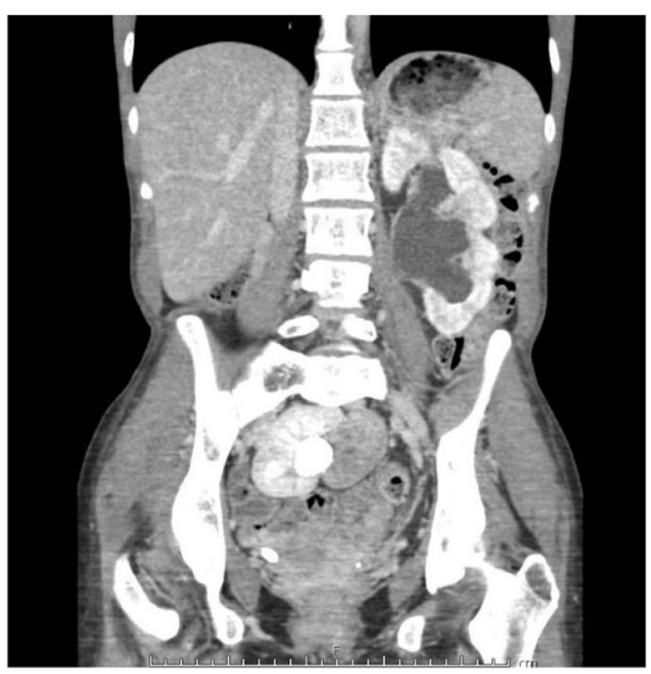

Fig. 1 CT urography image shows bilateral gross hydronephrosis with congenital pelvic right kidney 


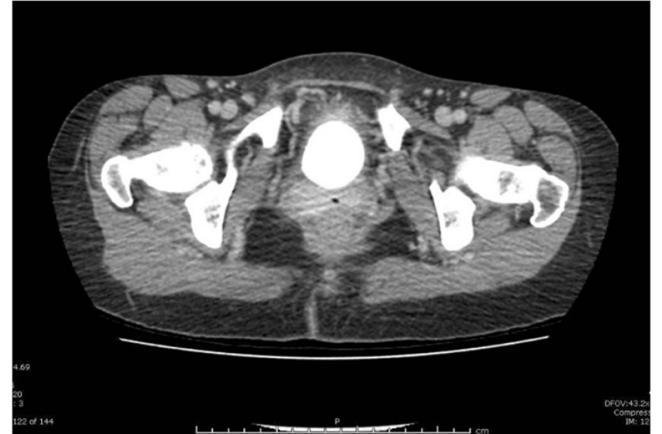

Fig. 2 CT urography image shows a small shrunken bladder with diffuse wall thickening

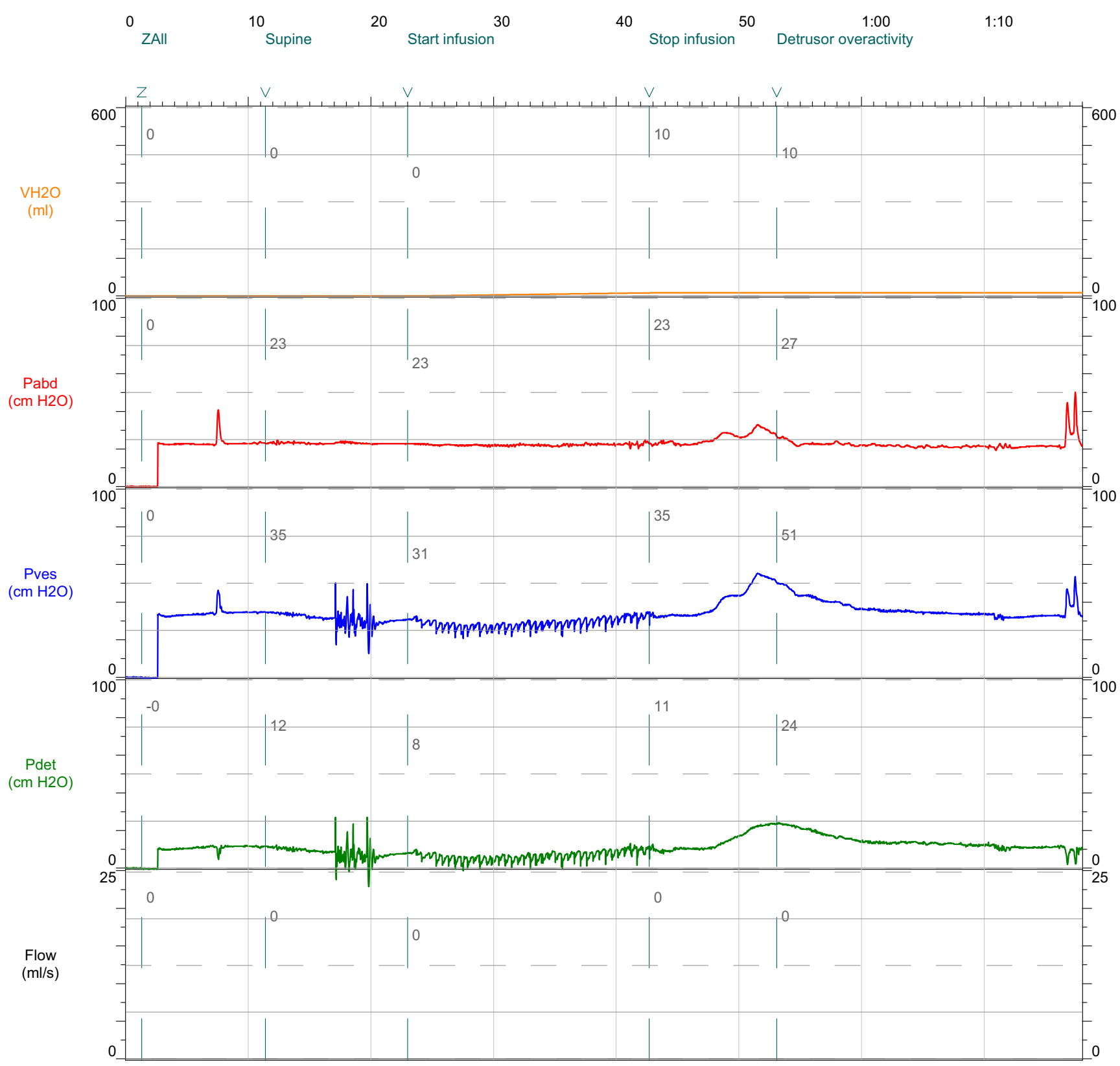

Fig. 3 Video urodynamic study (pressure flow against time plot) shows small bladder capacity $(<50 \mathrm{~mL})$, with associated detrusor overactivity reduced bladder capacity and never resume their function. The hypertonicity and irritability are greater in the congenital obstructed bladder when compared with the acquired obstruction [22]. Early intervention or obstruction repair in infants with congenital obstructive lesions avoids the complications of a contracted bladder later in life.

\section{Neurogenic Bladder}

The bladder can be altered differently in distinctive neurological conditions and at different stages. Several molecular pathways have been postulated in the development of neurogenic 
detrusor overactivity, detrusor underactivity and detrusor sphincter dyssynergia. Neurogenic bladders present altered tissue composition including an ICB [23]. Management for these conditions should be based on mechanisms to empty the bladder and protect the upper tract.

\section{Infections}

\section{Tuberculosis}

Tuberculosis (TB) is an infection caused by Mycobacterium tuberculosis; genitourinary affection is the second most common form of extrapulmonary TB following the lymph nodes. When Mycobacterium tuberculosis affects the urinary tract, it usually begins in the kidneys. Descending infection to the bladder usually spreads through the ureteral orifices and lymphatics. Bacilli cause ulcerations in the urothelium producing mucosal inflammation, haematuria and friability. It has been postulated that after a year of chronic inflammation produced by $\mathrm{TB}$, the mucosal scarring leads to bladder contracture with affectation of the upper tract and possibly urethral involvement [24]. Urinary diversion and bladder augmentation are treatment options for this form of IBC.

\section{Schistosomiasis}

This is a parasitic urinary tract infection caused by Schistosoma haematobium; these flatworms are released from snails and spread in water, then penetrate the human skin and develop in the lungs and liver. Once mature, these adult worms deposit their eggs in the bladder wall vessels and produce a granulomatous response provoking chronic inflammatory response and fibrosis. This form of ICB is a risk factor for squamous carcinoma of the bladder. Treatment options include praziquantel, but this antihelminthic has no effect on the morphologic changes of the bladder wall [25].

\section{Inflammatory Conditions}

\section{Interstitial Cystitis}

Although the taxonomy of this entity is generally described as bladder pain syndrome, some phenotypes present structural changes in the bladder wall that are compatible with the ICB. Traditionally, interstitial cystitis (IC) is described with Hunner lesions, small mucosa vessels radiating towards a central scar and/or the presence of glomerulations seen after hydrodistention. IC is associated with a reduced bladder capacity and it shares some of the pathophysiology pathways of the ICB $[26,27]$.

\section{Malacoplakia}

Malacoplakia is an inflammatory condition in which host defences and phagocytosis are altered. It affects several organs such as lungs, skin, mesenteric lymph nodes and gastrointestinal and urinary tracts. Malacoplakia is characterised by soft, yellow-brown plaques associated with Escherichia coli infections. A granulomatous reaction is produced containing histiocytes also known as Michaelis-Gutmann bodies [28]. When it affects the urinary tract, this condition can progress to ICB, especially when present with multifocal lesions [25]. The progression of this disease is associated with substantial morbidity and a mortality of more than $50 \%$.

Treatment of malacoplakia is mostly medical. In view of the fact that malacoplakia is a rare condition, no widely medical treatment guidelines have been established. Associated urinary tract infections can be treated with antibiotic [29] that are effective at intracellular level such as quinolones (mainstay of treatment), trimethoprim and rifampicin. These antibiotics are able to aid the defective phagolysosomal mechanism found in malakoplakia. These can be used long-term at low doses to prevent recurrence. Sometimes in advance malacoplakia, surgical intervention maybe indicated $[28,30]$. Operative procedure depends on the organ(s) affected. In cases where the lesion is causing obstruction to the ureters, transurethral resection can be beneficial. Nephrostomy or ureteric stenting may also be considered. In advanced disease which involves extensive pelvic malacoplakia, complex abdominal surgery which may involve bladder and bowel resection may be necessary especially if the bowel is involved.

\section{Eosinophilic Cystitis}

This inflammatory bladder condition is rare and is represented by fibrosis and inflammation in the bladder with or without muscle necrosis. This inflammation is postulated to be caused by antigen-antibody complexes which attract eosinophils to the bladder wall. This results in activation of enzymes and cytokines that triggers the development of the ICB [25]. Several aetiological factors have been documented for eosinophilic cystitis such as recurrent urinary tract infections, parasites, urinary tract surgeries, transitional cell carcinoma, chronic granulomatous disease, eosinophilic enteritis, ovarian teratoma, mitomycin $\mathrm{C}$, tranilast [31-33] (also known as Rizaben, is an antioxidant, antiallergic, antiangiogenesis and anti-inflammatory agent that is used to treat disorders ranging from bronchial asthma, keloids and oligozoospermia), and some foods such as tomatoes, carrots and coffee [34]. This condition could be self-limited; initial management consist of non-steroidal anti-inflammatory drugs (NSAIDs), anti-histamines, corticosteroids and cyclosporine. Cystectomy can be a treatment option when eosinophilic cystitis reaches the point of the ICB with hydronephrosis [34]. 


\section{Drugs and Medications}

\section{Ketamine}

The recreational use of ketamine, an anaesthetic agent that is often prescribed for chronic pain management [35], has been associated with lower urinary tract symptoms with a prevalence of 20-90\% among users. This drug causes cystitis with haematuria and a contracted bladder with a significantly reduced bladder capacity [36]. The cystoscopic findings include epithelial inflammation in the form of ulcerations, neovascularization, denudation and petechia. There is no single definitive management; various therapies have been proposed such as antibiotics, NSAIDs, steroids, pentosan polysulphate, anticholinergics, hydrodistension and bladder instillations with hyaluronic acid or urinary diversion.

\section{Bacillus Calmette-Guérin, Mitomycin $C$ and Epirubicin}

The use of intravesical agents as adjuvant treatments for nonmuscle-invasive bladder cancer have been related to reduced bladder capacity and contracted bladder in less than $5 \%$ of cases [37], mostly because of extravasation of intravesical therapy. This is a serious complication and is associated with multiple tumour resections and maintenance instillations. Intravesical immunotherapy or chemotherapy must be discontinued and a radical cystectomy should be considered as part of treatment $[38,39]$.

\section{Gefitinib}

Gefitinib is an oral chemotherapy that inhibits the epidermal growth factor receptor (EGFR) tyrosine kinase and is mostly used as part of the treatment for non-small-cell lung cancer. Some of the side effects of this medication include diarrhoea and skin rash; however, haemorrhagic cystitis and the development of ICB have been documented [40]. When patients on this therapy present with urinary symptoms, gefitinib should be halted.

\section{Radiation Cystitis}

Animal models have demonstrated that radiation to the bladder reduces cystometric capacity and compliance therefore causing bladder dysfunction. These models have failed to explain historical findings of urothelial injury and fibrosis [41]. New pelvic radiotherapy techniques have reduced the toxicity of this therapeutic modality; nonetheless, some side effects compatible with the ICB have been documented [25, 42].

\section{Chronic Use of Catheters}

Several studies have suggested that use of indwelling catheters on free drainage can contribute to a reduction of the bladder capacity and function. After a period of 6-12 months, the production of muscular fibrosis in the bladder wall leads to structural changes that interfere with volumetric capacity and compliance [43]. The use of valves in patients with indwelling catheters gives the opportunity to mimic the healthy bladder and has the potential to maintain, bladder capacity, tone and function [44].

\section{Discussion}

In the same way that Krotoszyner stated that the ICB can be related to several urological conditions, we suggest that some pathologies can develop an ICB-like presentation. We theorise that some pelvic tumours like muscle-invasive transitional cell carcinoma or advance prostate cancer, urinary fistula and patients with multiple bladder surgeries can show some of the morphologic features of the ICB. Krotoszyner presented a case of a patient with sarcoma of the bladder and a case of severe cystitis due to a bladder stone; both cases had ICB characteristics [2].

Although the most common aetiologies of the ICB have been presented in this article, other vesical pathologies can be associated with some of the characteristics of the ICB. A number of studies have suggested that ageing can lead to a reduced cystometric capacity [10]. Elderly patients with detrusor overactivity can present with a reduced bladder capacity as well [11]; however, these findings do not entirely correspond with the morphology of the ICB.

Animal experimentations which induced metabolic syndrome have shown the presence of bladder alterations such as hypoxia and fibrosis [45]; nonetheless, there is no evidence of ICB in humans with metabolic syndrome.

End-stage kidney disease patients who present with oliguria or anuria and are on dialysis can develop a reduced cystometric capacity and compliance; a mean volume of $178 \mathrm{~mL}$ has been documented with the lowest range of $14 \mathrm{~mL}$ [46].

Certain connective tissue pathologies like systemic sclerosis or scleroderma have been associated with lower urinary tract symptoms, urodynamic abnormalities and bladder wall fibrosis [47]; however; the lowest volumetric capacity was reported with $114 \mathrm{~mL}$.

\section{Conclusions}

The inflammatory contracted bladder (ICB) can be found in a wide spectrum of urological conditions. Moreover, some 
pathologies of the urinary tract can develop certain ICB morphologic characteristics. Assessment and management should be tailored according to the aetiology and stage of this vesical entity.

\section{Compliance with Ethical Standards}

Conflict of Interest The authors declare that they have no conflict of interest.

Human and Animal Rights and Informed Consent This article does not contain any studies with human or animal subjects performed by any of the authors.

Open Access This article is distributed under the terms of the Creative Commons Attribution 4.0 International License (http:// creativecommons.org/licenses/by/4.0/), which permits unrestricted use, distribution, and reproduction in any medium, provided you give appropriate credit to the original author(s) and the source, provide a link to the Creative Commons license, and indicate if changes were made.

\section{References}

Papers of particular interest, published recently, have been highlighted as:

- Of importance

-• Of major importance

1. Leçons cliniques sur les maladies des voies urinaires : sémiologie, diagnostic, pathologie et thérapeutique générales : professées à l'hôpital Necker. Symptômes fonctionnels, modifications pathologiques des urines / par J.-C.-Félix Guyon,... Gallica. https://gallica.bnf.fr/ark:/12148/bpt6k5663156r/f6.image. texteImage. Accessed 6 Nov 2018.

2. Krotoszyner M. The Cystoscopic diagnosis of contracted bladder. J Am Med Assoc. 1917;LXVIII(9):687. https://doi.org/10.1001/ jama.1917.04270030019006.

3.• Abrams P, Cardozo L, Fall M, et al. The standardisation of terminology of lower urinary tract function: report from the standardisation sub-committee of the international continence society. Neurourol Urodyn. 2002;21(2):167-78. https://doi.org/10. 1002/nau.10052 Describes the terminology of the urinary tract.

4. Lee Y, Lin K, Chuang S, et al. Elucidating mechanisms of bladder repair after hyaluronan instillation in ketamine-induced ulcerative cystitis in animal model. Am J Pathol. 2017;187(9):1945-59. https://doi.org/10.1016/j.ajpath.2017.06.004.

5. Fry C. Ics.org. https://www.ics.org/Publications/ICI 3/v1.pdf/ chap6.pdf. Published 2018. Accessed 6 Nov 2018. Highlights the cellular mechanism of the urinary tract.

6. Kaplan E, Richier J, Howard P, Ewalt D, Lin V. Type III collagen messenger RNA is modulated in non-compliant human bladder tissue. J Urol. 1997;157(6):2366-9. https://doi.org/10.1016/s00225347(01)64782-7.

7. Djavan B, Lin V, Kaplan E, et al. Decreased elastin gene expression in noncompliant human bladder tissue: a competitive reverse transcriptase-polymerase chain reaction analysis. J Urol. 1998;160(5):1658-62. https://doi.org/10.1016/s0022-5347(01) 62375-9.

8. Zhang E, Stein R, Chang S, et al. Smooth muscle hypertrophy following partial bladder outlet obstruction is associated with overexpression of non-muscle Caldesmon. Am J Pathol. 2004;164(2):601-12. https://doi.org/10.1016/s0002-9440(10) 63149-5.

9. Dmochowski RR. Bladder outlet obstruction: etiology and evaluation. Rev Urol. 2005;7(Suppl 6):S3-S13.

10. Madersbacher S, Pycha A, Schatzl G, Mian C, Klingler C, Marberger M. The aging lower urinary tract: a comparative urodynamic study of men and women. Urology. 1998;51(2):20612. https://doi.org/10.1016/s0090-4295(97)00616-x.

11. Pfisterer M, Griffiths D, Schaefer W, Resnick N. The effect of age on lower urinary tract function: a study in women. J Am Geriatr Soc. 2006;54(3):405-12. https://doi.org/10.1111/j.1532-5415. 2005.00613.x.

12. Rakesh K, Ansari MS, Anil M, Anil G. Clinical presentation and diagnostic approach in cases of genitourinary tuberculosis. Indian J Urol. 2008;24(3):401-5.

13. Zarrabi AD, Heyns CF. Clinical features of confirmed versus suspected urogenital tuberculosis in region with extremely high prevalence of pulmonary tuberculosis. Urology. 2009;74(1):41-5.

14. Kao SC, Fang JT, Tsai CJ, Chen KS, Huang CC. Urinary tract tuberculosis: a 10-year experience. Changgeng Yi Xue Za Zhi. 1996;19(1):1-9.

15. Kulchavenya E. Best practice in the diagnosis and management of urogenital tuberculosis. Ther Adv Urol. 2013;5(3):143-51.

16. Ross AG, Bartley PB, Sleigh AC, Olds GR, Li Y, Williams GM, et al. Schistosomiasis. N Engl J Med. 2002;346(16):1212-20.

17. Gryseels B, Polman K, Clerinx J, Kestens L. Human schistosomiaisis. Lancet. 2006;368(9541):1106-18.

18. Winstock AR, Mitcheson L, Gillatt DA, Cottrell AM. The prevalence and natural history of urinary symptoms among recreational ketamine users. BJU Int. 2012;110:1762-6.

19. Middela S, Pearce I. Ketamine-induced vesicopathy: a literature review. Int J Clin Pract. 2011;65(1):27-30.

20. Chu PS, Ma WK, Wong SC, Chu RW, Cheng CH, Wong S, et al. The destruction of the lower urinary tract by ketamine abuse: a new syndrome? BJU Int. 2008;102(11):1616-22.

21. Tsai TH, Cha TL, Lin CM, Tsao CW, Tang SH, Chuang FP, et al. Ketamine-associated bladder dysfunction. Int J Urol. 2009;16(10): 826-9.

22. Tanagho E. Congenitally obstructed bladders: fate after prolonged defunctionalization. J Urol. 1974;111(1):102-9. https://doi.org/10. 1016/s0022-5347(17)59900-0.

23. Nagatomi J, DeMiguel F, Torimoto K, Chancellor M, Getzenberg $\mathrm{R}$, Sacks M. Early molecular-level changes in rat bladder wall tissue following spinal cord injury. Biochem Biophys Res Commun. 2005;334(4):1159-64. https://doi.org/10.1016/j.bbrc.2005.07.011.

24. Singh O, Gupta S, Arvind N. A case of extensive genitourinary tuberculosis: combined augmentation ileo-cystoplasty, ureteric ileal replacement and buccal mucosal graft urethroplasty. Updat Surg. 2012;65(3):245-8. https://doi.org/10.1007/s13304-012-0141-8.

25. Wong-You-Cheong J, Woodward P, Manning M, Davis C. Inflammatory and nonneoplastic bladder masses: radiologicpathologic correlation. RadioGraphics. 2006;26(6):1847-68. https://doi.org/10.1148/rg.266065126.

26. Awad S, MacDiarmid S, Gajewski J, Gupta R. Idiopathic reduced bladder storage versus interstitial cystitis. J Urol. 1992;148(5): 1409-12. https://doi.org/10.1016/s0022-5347(17)36923-9.

27. Fall M, Johansson S, Aldenborg F. Chronic interstitial cystitis: a heterogeneous syndrome. J Urol. 1987;137(1):35-8. https://doi. org/10.1016/s0022-5347(17)43863-8.

28. Wein A, Kavoussi L, Partin A, Peters C, Campbell M. CampbellWalsh Urology. 11th ed. Amsterdam: Elsevier; 2016. p. 289.

29. Dasgupta P, Womack C, Turner A. Malacoplakia: Von Hansemann's disease. BJU Int. 1999;84:464-9. 
30. Huan D, Sarah D, Joe P, Shalini C, Kesavapilla S. Malacoplakia of the urogenital tract. Urol Case Rep. 2015;3(1):6-8. https://doi.org/ 10.1016/j.eucr.2014.10.002.

31. Vail J. Innovative uses of compound tranilast: an interview with compounding pharmacist Larry J. Frieders, RPh. Int J Pharm Compd. 2007;11(2):130-3.

32. Okada H, Minayoshi K, Goto A. Two cases of eosinophilic cystitis induced by tranilast. J Urol. 1992;147(5):1366-8.

33. Sakai N, Yamada T, Murayama T. Eosinophilic cystitis induced by tranilast: a case report. Hinyokika Kiyo. 1998;44(1):45-7.

34. Teegavarapu P, Sahai A, Chandra A, Dasgupta P, Khan M. Eosinophilic cystitis and its management. Int J Clin Pract. 2005;59(3):356-60. https://doi.org/10.1111/j.1742-1241.2004. 00421.x.

35. Middela S, Pearce I. Ketamine-induced vesicopathy: a literature review. Int J Clin Pract. 2010;65(1):27-30. https://doi.org/10. 1111/j.1742-1241.2010.02502.x.

36. Srirangam S, Mercer J. Ketamine bladder syndrome: an important differential diagnosis when assessing a patient with persistent lower urinary tract symptoms. BMJ Case Rep. 2012;2012. https://doi.org/ 10.1136/bcr-2012-006447.

37. O'Donnell M. Practical applications of Intravesical chemotherapy and immunotherapy in high-risk patients with superficial bladder cancer. Urol Clin N Am. 2005;32(2):121-31. https://doi.org/10. 1016/j.ucl.2005.01.003.

38. Witjes J, Palou J, Soloway M, et al. Clinical practice recommendations for the prevention and management of intravesical therapyassociated adverse events. Eur Urol Suppl. 2008;7(10):667-74. https://doi.org/10.1016/j.eursup.2008.08.001.

39. Wajsman Z, McGill W, Englander L, Huben R, Pontes J. Severely contracted bladder following intravesical mitomycin $\mathrm{C}$ therapy. J Urol. 1983;130(2):340-1. https://doi.org/10.1016/s0022-5347(17) 51139-8.

40. Arakawa M, Nakamura K, Yamada Y, Rosser CJ, Tobiume M, Saito $\mathrm{H}$, et al. Association between gefitinib and hemorrhagic cystitis and severely contracted bladder: a case report. BMC Urol. 2010;10(1). https://doi.org/10.1186/1471-2490-10-6.

41. Vale J, Bowsher W, Liu K, Tomlinson A, Whitfield H, Trott K. Post-irradiation bladder dysfunction: development of a rat model. Urol Res. 1993;21(6):383-8. https://doi.org/10.1007/bf00300073.

42. Huang W, Yang J. Sonographic findings in a case of postradiation hemorrhagic cystitis resolved by hyperbaric oxygen therapy. J Ultrasound Med. 2003;22(9):967-71. https://doi.org/10.7863/jum. 2003.22.9.967.

43. Kristiansen P, Pompeius R, Wadström L. Long-term urethral catheter drainage and bladder capacity. Neurourol Urodyn. 1983;2(2): 135-43. https://doi.org/10.1002/nau.1930020207.

44. Virdi G, Hendry D. Urinary retention: catheter drainage bag or catheter valve? Curr Urol. 2015;9(1):28-30. https://doi.org/10. $1159 / 000442847$.

45. Morelli A, Comeglio P, Filippi S, Sarchielli E, Cellai I, Vignozzi L, et al. Testosterone and farnesoid X receptor agonist INT-747 counteract high fat diet-induced bladder alterations in a rabbit model of metabolic syndrome. J Steroid Biochem Mol Biol. 2012;132(1-2): 80-92. https://doi.org/10.1016/j.jsbmb.2012.02.007.

46. Chen J, Lee M, Kuo H. Reduction of cystometric bladder capacity and bladder compliance with time in patients with end-stage renal disease. J Formos Med Assoc. 2012;111(4):209-13. https://doi.org/ 10.1016/j.jfma.2011.09.023.

47. Minervini R, Morelli G, Minervini A, Pampaloni S, Tognetti A, Fiorentini L, et al. Bladder involvement in systemic sclerosis: urodynamic and histological evaluation in 23 patients. Eur Urol. 1998;34(1):47-52. https://doi.org/10.1159/000019678.

Publisher's Note Springer Nature remains neutral with regard to jurisdictional claims in published maps and institutional affiliations. 